\title{
EXOTIC SINGULAR STRUCTURES ON SPHERES
}

\author{
BY
}

\author{
NORMAN LEVITT $\left(^{1}\right)$
}

\begin{abstract}
It is shown how the category of $P L$-manifolds may be obtained from the smooth category by an iterative procedure, viz., first form singular smooth manifolds where smooth seven-spheres are allowed as links. Then, in the new category one has obtained, kill all eight-spheres in similar fashion. Repeating this process ad infinitum (but requiring only finitely many stages in each dimension), one obtains the category of $P L$-manifolds. By taking care that the set of "singular" points is always given enough structure, it is seen that this iterative process corresponds to a skeletal filtration of $B P L \bmod B O$. Also, a geometric interpretation of the Hurewicz map $\pi_{*}(B P L, B O) \rightarrow H_{*}(B P L, B O)$ is inferred.
\end{abstract}

1. Introduction. The object of this paper is to re-examine the differences between smooth and $P L$-manifolds via the geometry of $P L$-manifolds whose failure to be smooth may be measured, in some sense, by singularities. The germinal idea is this: From the ideas of Sullivan [a] and Baas [b] we know how to talk about a manifold with singularities; that is, a space which is of the form

$$
M^{n}=M_{0}^{n} \cup_{\partial M_{0}} S
$$

where $M_{0}^{n}$ is an $n$-manifold with boundary, $S$ is of the form $P^{n-r} \times c Q^{r-1}$, with $P^{n-r}, Q^{r-1}$ manifolds, $\partial M_{0}^{n}=P^{n-r} \times Q^{r-1}$ and $c$ denotes unreduced cone.

Now if $M_{0}^{n}, P^{n-r}$ are smooth manifolds and $Q^{r-1}=\Sigma^{r-1}$ is a smoothness structure on the $P L(r-1)$ sphere, we see that $M^{n}$ is a $P L$-manifold where lack of smoothness resides in the "singular" structure in a neighborhood of $P^{n-r}$. We can iterate this process, i.e., we can find all $P L$-manifolds constructed in this way, using a certain set of exotic spheres as "allowable" singularities, and then go looking for exotic structures on spheres in this "new" category. We then use some of these spheres as singularities to create yet another category of singular manifolds (and so on), noting the important fact that at every stage the singular manifolds which we introduce retain underlying $P L$-manifold structures.

The natural question, of course, is: does this process terminate? That is, do

Received by the editors March 22, 1974.

AMS (MOS) subject classifications (1970). Primary 57C25; Secondary 57C20, 57D60.

Key words and phrases. Manifold-like category, singular manifold, exotic structures on spheres, thickenings.

(1) The author was partially supported by NSF grant GP-43827. 
we ever reach the $P L$-manifold category in this way, at least in the limit?

The answer is "yes" and, moreover, the process of proving this is closely connected with the construction of a certain relative cell structure from $B O$ to $B P L$. In addition, we obtain a nice interpretation of the Hurewicz homomorphism

$$
h: \pi_{n}(B P L, B O) \rightarrow H_{n}(B P L, B O) .
$$

In particular, $H_{n}(B P L, B O)$ is viewed as a group of equivalence classes of certain kinds of singular structures on $S^{n-1}$, where equivalence is given by a certain kind of concordance and the group operation is connected sum. The Hurewicz homomorphism is then seen to be the natural forgetful map from the group of smooth structures on $S^{n-1}=\pi_{n-1}(P L / O)=\pi_{n}(B P L, B O)$.

A final word about what the category theorists will quite properly regard as an abuse of language. We use terms like "category of smooth manifolds" and "category of $P L$-manifolds" to denote the entities which topologists have in mind when they make informal use of these terms. We also make a more formal definition (see below) of something which we call a "manifold-like category." The word "category" in this context should by no means be equated with the objectmorphism gadget of formal category-theory.

2. Manifold-like categories. In this section, we shall formalize, to a certain extent, the notion of an "additional structure" on a $P L$-manifold. In particular, we have in mind a formalism which is a rubric, of sorts, for the naive idea of additional geometric structure, as opposed to the conventional notion that a structure shall be defined as a certain reduction of the $P L$-tangent bundle. In fact, one of the purposes of this formalism is to set up conditions for geometric structures which enable existence and uniqueness questions to be transformed into bundle reduction or lifting problems.

We shall define the notion of a manifold-like category $C$. $C$ is, first of all, a set whose elements are called $C$-manifolds. Intuitively, a $C$-manifold is to be thought of as a $P L$-manifold together with an additional structure. A good example to keep in mind (the paradigm, in fact, for this construction) is the category of smooth structures on $P L$-manifolds.

We axiomatize the properties of a manifold-like category as follows:

A-(1). For each $C$-manifold $M^{n}$, there is a unique $P L$-manifold $M_{P L}^{n}$. The dimension of $M^{n}$ is $n$. There is a dimension-lowering boundary operation $\partial$ so that $\left(\partial M^{n}\right)_{P L}=\partial\left(M_{P L}^{n}\right)$. There is a dimension-preserving involution $\iota$ on $C$-manifolds which commutes with $\partial$. Moreover $\left(L M^{n}\right)_{P L}=M_{P L}^{n}$.

A-(2). If $P^{n}$ is a smoothing of a $P L$-manifold $Q^{n}$ then $P^{n}$ determines a unique $C$-manifold $C\left(P^{n}\right)$ with $\left(C\left(P^{n}\right)\right)_{P L}=Q^{n}$. Moreover, $C\left(\partial P^{n}\right)=\partial C\left(P^{n}\right)$ and $\iota C\left(P^{n}\right)=C\left(P^{n}\right)$. Moreover there is a unique $C$-manifold $\phi^{n}$ with $\phi_{P L}^{n}=\varnothing$. 
A-(3). (a) If $M^{n}, N^{n}$ are $C$-manifolds, then there is an operation of disjoint union in $C$, denoted $\amalg$ with $\left(M^{n} \amalg N^{n}\right)_{P L}=M_{P L}^{n} \amalg N_{P L}^{n}$.

(b) If $M^{n}, N^{n}$ are $C$-manifolds with $\partial M^{n}=\iota \partial N^{n}$, there is an operation of union along boundary in $C$, denoted $\cup_{\partial}$ such that $\left(M^{n} \cup_{\partial} N^{n}\right)_{P L}=M_{P L}^{n} \cup_{\partial} N_{P L}^{n}$.

(c) Let $M^{n}, N^{n}$ be $C$-manifolds with

$$
\partial M^{n}=Q^{n-1} \cup_{\partial} P^{n-1}, \quad \partial N^{n}=\imath Q^{n-1} \cup_{\partial} R^{n-1} .
$$

Then we may form $M^{n} \cup_{\underline{\partial}} N^{n}$ with

$$
\left(M^{n} \cup_{\underline{\partial}} N^{n}\right)_{P L}=M_{P L}^{n} \cup_{Q_{P L}^{n-1}} N_{P L}^{n} .
$$

All the above union operations commute with $\iota$.

A-(4). There is an operation $I \times$ such that $\left(I \times M^{n}\right)_{P L}=I \times M_{P L}^{n}$. (Here, the $I$ on the right-hand side is the standard unit interval and $\times$ denotes usual cartesian product.) If $P^{n}$ is a smoothing of a $P L$-manifold $I \times C\left(P^{n}\right)=C\left(I \times P^{n}\right)$. $\partial\left(I \times M^{n}\right)=\left(M^{n} \amalg \iota M^{n}\right) \cup_{\partial}\left(I \times \partial M^{n}\right)$. Finally $\iota\left(I \times M^{n}\right)=I \times \iota M^{n}$. We define $D^{m} \times$ as the operation $(I \times)^{m}$.

We pause here for a definition.

2.1. Definition. Two $C$-manifolds $M^{n}, N^{n}$ are said to be concordant iff there is a $C$-manifold $W^{n+1}$ with $\partial W^{n+1}=\left(M^{n} \amalg \iota N^{n}\right) \cup_{\partial} V^{n}$ such that

and

$$
W_{P L}^{n+1} \cong I \times M_{P L}^{n} \cong I \times N_{P L}^{n}
$$

$$
V_{P L}^{n} \cong I \times \partial M_{P L}^{n} \cong I \times \partial N_{P L}^{n} \text {. }
$$

A-(5) (Regular Neighborhood Property). Let $M^{n}$ be a $C$-manifold and let $K$ be a subcomplex of the interior of the $P L$-manifold $M_{P L}^{n}$. Then $M^{n}$ is concordant to $N^{n}$ such that $N^{n}=P^{n} \cup_{\partial} Q^{n}$ where $P_{P L}^{n}$ is a $P L$-regular neighborhood of $K$. (The union of $P^{n}$ and $Q^{n}$ is along $\partial P^{n}$.) Moreover, if $\bar{N}^{n}=\bar{P}^{n} \cup_{\partial} \bar{Q}^{n}$ is another $C$-manifold concordant to $M^{n}$ with $\bar{P}_{P L}^{n}$ a regular neighborhood of $K$, then there is a concordance $W^{n+1}$ between $N^{n}$ and $\bar{N}^{n}$, which is the union of concordances $A^{n+1}$ between $P^{n}$ and $\bar{P}^{n}$ and $B^{n+1}$ between $Q^{n}$ and $\bar{Q}^{n}$ (the union being taken along $C^{n}$, a concordance between $\partial P^{n}$ and $\partial \bar{P}^{n}$ ), and subject to the condition $I \times K \subseteq A_{P L}^{n+1}$. Furthermore, a relative version of the above holds if $K, L$ is a simplicial pair contained in $M_{P L}^{n}, \partial M_{P L}^{n}$.

A-(6) (Cerf Property). If $M^{n}$ is a $C$-manifold, $M^{n}$ is concordant to $M_{0}^{n} \cup$ $C\left(D^{n}\right)$ where $D^{n}$ is the standard smooth $n$-disc and the union is along $C\left(S^{n-1}\right)$. Moreover, this decomposition is unique up to concordance. That is, if $D_{1}^{n}, D_{2}^{n}$ are two copies of the smooth disc and $M_{1}^{n}=P_{1}^{n} \cup_{\partial} C\left(D_{1}^{n}\right)$ is concordant to $M_{2}^{n}=$ $P_{2}^{n} \cup_{\partial} C\left(D_{2}^{n}\right)$, then there is a concordance $W^{n+1}$ between $M_{1}^{n}$ and $M_{2}^{n}$ such that $W^{n+1}=Q^{n+1} \cup C\left(E^{n+1}\right)$ where $E^{n+1}$ is a smooth concordance between $D_{1}^{n}$ and $D_{2}^{n}$. 
REMARK. It follows from A-(6) that it will be possible to define connected sum for $C$-manifolds.

2.2. Definition. A $C$-structure on a $P L$-manifold $X^{n}$ is a $C$-manifold $M^{n}$ together with a $P L$ homeomorphism $h: M_{P L}^{n} \rightarrow X^{n}$. If $\left(M^{n}, h\right),\left(N^{n}, g\right)$ are two $C$-structures on $A^{n}$ they are said to be concordant iff there is a $C$-structure $\left(W^{n+1}, F\right)$ on $I \times X^{n}$ with $\partial W^{n+1}=\left(M^{n} \amalg \iota N^{n}\right) \cup V^{n}$ where $V^{n}$ is a concordance between $\partial M^{n}$ and $\partial N^{n}$ where $h, g$ are given respectively by

$$
\begin{aligned}
& M_{P L}^{n} \subseteq W_{P L}^{n+1} \stackrel{F}{\rightarrow} I \times X \stackrel{\text { proj }}{\rightarrow} X^{n}, \\
& N_{P L}^{n} \subseteq W_{P L}^{n+1} \stackrel{F}{\rightarrow} I \times X^{n} \stackrel{\text { proj }}{\rightarrow} X^{n} .
\end{aligned}
$$

Concordance is obviously an equivalence relation on $C$-structures. Let $S_{C}\left(X^{n}\right)$ be the set of concordance classes of $C$-structures on $X^{n}$.

REMARK. There is an obvious map $S_{C}\left(X^{n}\right) \rightarrow S_{C}\left(I \times X^{n}\right)$. Furthermore, $S_{C}\left(S^{n}\right)$ becomes an abelian group by virtue of Axiom (6). We call this group $\theta_{C}^{n}$. There is a forgetful homomorphism $\theta^{n} \rightarrow \theta_{C}^{n}$ where $\theta^{n}$ is the usual group of smoothings of $S^{n}$. Let $\left[\left(\Sigma^{n}, h\right)\right]$ be an element of $S_{C}\left(S^{n}\right)$ where $h: \Sigma_{P L}^{n} \rightarrow S^{n}$. We describe $-\left[\left(\Sigma^{n}, h\right)\right]$ as follows: By Axiom (4), $\partial\left(I \times \Sigma^{n}\right)=\Sigma^{n} \amalg \iota \Sigma^{n}$. So there is an obvious homeomorphism $\iota \Sigma_{P L}^{n} \subseteq\left(I \times \Sigma^{n}\right)_{P L} \stackrel{\text { proj }}{\longrightarrow} \Sigma_{P L}^{n} \stackrel{h}{\rightarrow} S^{n}$. Let $g$ be the composition of this homeomorphism with the homeomorphism $S^{n} \rightarrow$ $S^{n}$ given by the matrix $\left[\begin{array}{cc}-1 & 0 \\ 0 & I\end{array}\right]$ in $R^{n+1}$. The formula is - [( $\left.\left.\Sigma^{n}, h\right)\right]=\left[\left(\iota \Sigma^{n}, g\right)\right]$.

There are two important potential properties of a manifold-like category which we wish to examine with some care.

Property (I). Let $M^{n+1}$ be a $C$-manifold satisfying

(i) $M_{P L}^{n+1} \doteq I \times X^{n}$.

(ii) $\partial M^{n+1}=\left(I \times P^{n-1}\right) \cup_{\partial} Q^{n}$ where $P^{n-1}$ is a $C$-manifold having $P_{P L}^{n-1}$ a codimension 0 submanifold of $\{0\} \times \partial X^{n}$.

Then there is a $C$-manifold $W^{n+2}$ satisfying

(a) $W_{P L}^{n+2}=I \times M_{P L}^{n+1}$.

(b) $\partial W^{n+2}=M^{n+1} U_{\partial} A^{n+1} U_{\underline{\partial}}\left(I \times V^{n}\right)$ where $A^{n+1}, V^{n}$ are $C$-manifolds satisfying:

$$
A_{P L}^{n+1}=\stackrel{0}{I} \times \partial M_{P L}^{n+1}, \quad V_{P L}^{n}=\{(0,1)\} \times X^{n},
$$

and where $A^{n+1}=B^{n+1} \cup_{\underline{\partial}} C^{n+1}$ with $B^{n+1}=I \times I \times P^{n-1}$.

What Property (I) asserts is, briefly, this: If the $P L$-manifold $X^{n} \times I$ should happen to have a $C$-structure, then this $C$-structure is deformable to one coming from a $C$-structure on $X^{n}$ via the operation $I \times$; moreover, this deformation can be kept constant along a codimension 0 piece of $\partial\left(X^{n} \times I\right)$ corresponding to a codimension 0 piece of $\partial X^{n}$ which has already been given a $C$-structure $P^{n-1}$, 
the assumption being that $I \times P^{n-1}$ fits into the given $C$-structure on $I \times X^{n}$.

Property (I) is modeled on an important property of smoothings of $P L$-manifolds [c], [d].

Property (II). Let $\Sigma^{n}$ be a $C$-manifold with $\Sigma_{P L}^{n} \cong S^{n}$. Then there is a $C$-manifold $\Delta^{n+1}$ with $\partial \Delta^{n+1}=\Sigma^{n}$ and $\Delta_{P L}^{n+1} \cong D^{n+1}$.

Property (II) says, in effect, that there are no "exotic" spheres in the manifold-like category $C$.

2.3. Lemma. If the manifold-like category $C$ satisfies Properties (I) and (II), then $C$ is the PL-manifold category. That is, given a PL-manifold $X^{n}$, $S_{C}\left(X^{n}\right)$ contains one and only one element.

Proof. The proof proceeds via handlebody theory [e]. We shall prove in detail only existence, i.e., that $S_{C}\left(X^{n}\right)$ is nonvoid. Uniqueness follows from an easy relativization of this proof.

Suppose, then, the existence assertion is true for $P L n$-manifolds admitting a handlebody decomposition where the highest dimensional handle is of dimension $k-1<n$. (Clearly, for $k-1=0$, this holds, thereby starting the induction.) Let $X^{n}$ be a $P L$-manifold with a $P L$-handle decomposition having handles of dimension no higher than $k$. For the sake of convenience we assume that $X^{n}$ has but one $k$-handle (the argument for more such handles involves mere repetition). By the inductive hypothesis, we assume that $X^{n}=X_{0}^{n} \cup(k$-handle) where $X_{0}^{n}=M_{P L}^{n}$ for some $C$-manifold $M^{n}$. Let $S^{k-1} \subseteq \partial X_{0}^{n}$ be the core sphere of the $k$-handle. By the Regular Neighborhood Axiom (5), we may as well assume that $\partial M^{n}=P^{n-1} \cup_{\partial} R^{n-1}$ where $R_{P L}^{n-1}$ is a regular neighborhood of $S^{k-1}$ (which has a certain product $P L$-structure $S^{k-1} \times D^{n-k}$ ). Thus $R^{n-1}$ determines a $C$-structure on $S^{k-1} \times D^{n-k}$. By repeated application of Property (I), we may as well assume $R^{n-1}=D^{n-k} \times \Sigma^{k-1}$ for some $C$-manifold $\Sigma^{k-1}$ with $\Sigma_{P L}^{k-1} \cong S^{k-1}$ and that the product structure $R_{P L}^{n-1}=S^{k-1} \times D^{n-k}$ thereby obtained is isotopic to the original one. Now, by Property (II), $\Sigma^{k-1}=\partial \Delta^{k}$ where $\Delta_{P L}^{k} \cong D^{k}$. Thus we may form $N^{n}=M^{n} \cup_{\underline{\partial}}\left(D^{n-k} \times \iota \Delta^{k}\right)$ where the union is along $D^{n-k} \times \Sigma^{k-1}=R^{n-1}$. Obviously $N_{P L}^{n}=X_{0}^{n} \cup(k$-handle $)=X^{n}$.

This proves that $X^{n}$ always admits a $C$-structure. That such a structure is always unique up to concordance will follow from an easy relative version of this argument, the details of which are left to the reader.

3. Killing exotic spheres. We shall now construct a sequence of manifoldlike categories which begins with the smooth category and converges to the category of $P L$-manifolds. Each of the intermediate categories will satisfy the Axioms (1)-(6) and Lemma 2.3 will be used to demonstrate that their limit is, indeed, the category of $P L$-manifolds. 
We begin, as we have noted, with the smooth manifolds. Consider the first dimension (7, as is well known [f] ) in which there is nonstandard smooth structure on the standard $P L$-sphere. We construct a manifold-like category $C_{7}$ as follows. First, pick one smooth structure on $S^{7}$ from each concordance class.

3.1. Definition. An $n$-dimensional $C_{7}$-manifold will consist of a smooth, compact manifold-with-boundary $M_{0}^{n}$, a finite set of compact smooth framed manifolds $V_{i}^{n-8}$ and a set of diffeomorphisms $h_{i}: U_{i}^{n-1} \rightarrow V_{i}^{n-8} \times \Sigma_{i}^{7}$ where the $U_{i}$ are disjoint, codimension 0 submanifolds of $\partial M_{0}^{n}$ and $\Sigma_{i}^{7}$ is one of the permissible smooth structures on $S^{7}$.

If $M^{n}$ is given by $M_{0}^{n}, V_{i}, h_{i}$ then $M_{P L}^{n}$ arises from the obvious construction, viz.

$$
M_{P L}^{n}=M_{0}^{n} \cup_{h_{i}} \cup\left(V_{i} \times c \Sigma_{i}^{7}\right)
$$

where $c$ denotes cones. The operations $\partial, I \times$ have the obvious meanings. We define the involution $\iota$ as follows: If $V_{i}^{n-8}$ is one of the framed manifolds specified in the definition, we alter $V_{i}^{n-8}$ to $W_{i}^{n-8}$ where $W_{i}^{n-8}$ has the same underlying smooth manifold and is framed by changing the framing of the normal bundle of $V_{i}^{n-8}$ by the constant matrix [ [ $\left.\begin{array}{rl}-1 & 0 \\ 0 & 1\end{array}\right]$.

We assert that $C_{7}$ is a manifold-like category, i. e., it satisfies Axioms (1)(6) of §2. The verification of most of these points is obvious and will therefore be left to the reader. The only marginally nontrivial axiom to establish for $C_{7}$ is the Regular Neighborhood Axiom (5). Let $K$ be a subcomplex of $M_{P L}^{n}$ for some $C_{7}$-manifold $M^{n}$. If we allow $K$ to be moved by an ambient isotopy, it is then easy to see that $K$ may be assumed to be in "general position" with respect to "singularities" $V_{i}$. That is,

$$
K=L \cup_{i}\left(\bigcup_{M_{i}} \times c \Sigma_{i}^{7}\right)
$$

where $M_{i}=K \cap V_{i}$ and $L=K \cap M_{0}^{n}$ with

$$
L \cap\left(\bigcup_{i} V_{i} \times \Sigma_{i}^{7}\right)=\bigcup_{i} M_{i} \times c \Sigma_{i}^{7} .
$$

Let $R=\bigcup_{i} R_{i}$ be a smooth regular neighborhood of $M=\bigcup_{i} M_{i}$ in $V=\bigcup_{i} V_{i}$. Then $\bigcup_{i}\left(R_{i} \times c \Sigma_{i}^{7}\right)$ is a regular neighborhood of $\bigcup_{i}\left(M_{i} \times c \Sigma_{i}^{7}\right)$ in $U_{i}\left(V_{i} \times c \Sigma_{i}^{7}\right)$, and $U_{i}\left(R_{i} \times \Sigma_{i}^{7}\right)=T_{1}$ is a smooth regular neighborhood of $\bigcup_{i}\left(M_{i} \times \Sigma_{i}^{7}\right)$. Extend $T_{1}$ to a smooth regular neighborhood $T$ of $L$ in $M_{0}^{n}$. Then $T \cup \cup_{i}\left(R_{i} \times c \Sigma_{i}^{7}\right)$ is a $P L$-regular neighborhood of $K$ which acquires an obvious $C_{7}$-manifold structure. This proves the existence part of Axiom (5). The uniqueness follows from a relative version of the same argument.

REMARK. It should have been apparent to the reader that we could well have defined a $C_{7}$-manifold differently and, at first glance, more simply, by 
merely specifying that the "singularities" $V_{i}^{n-8}$ of a $C_{7}$-manifold $M^{n}$ be ordinary smooth manifolds, rather than smooth manifolds with the seemingly superfluous additional structure of a framed normal bundle. In that case, the involution $\iota$ would have been trivial, i.e., the identity. Every result that we prove in this section for $C_{7}$, as defined above, could be proved with equal ease for $C_{7}$ with this simpler definition. However, the reasons for using the definition with the extra complication of framings on the singularity will emerge in $\S 4$.

The most important characteristic of $C_{7}$, beyond satisfying the axioms for a manifold-like category, is given in the following:

\subsection{Lemma. $C_{7}$ satisfies Property (I).}

Proof. We shall prove this in the absolute case, i.e., we consider a $C_{7^{-}}$. manifold $W^{n+1}$ where $W^{n+1}=I \times X^{n}$ without trying to keep track of some $C_{7}$-structure on a piece of $\partial X^{n}$. The reader should easily ascertain that introducing this complication, while lengthening the details of the proof, will in no way introduce any new essential difficulty.

Our problem, then, is to put a $C_{7}$-structure on $I \times W_{P L}^{n+1}$ which is the given one on $\{0\} \times W_{P L}^{n+1}$ and which is of the form $I \times M^{n}$ on $\{1\} \times W_{P L}^{n+1}$ where $M_{P L}^{n}=(1,0) \times X^{n}$.

First specify that $W_{P L}^{n+1}=W_{0}^{n+1} \cup \bigcup_{i}\left(V_{i}^{n-7} \times c \Sigma_{i}^{7}\right)$ where $W_{0}^{n+1}$ has a certain smoothing and $V_{i}^{n-8}$ is a smooth framed manifold. We identify $X^{n}$ with $\{0\} \times X^{n} \subseteq I \times X^{n}=W_{P L}^{n+1}$ and assume that $X^{n}$ is in general position with respect to $V_{i}^{n-7}$. (If not, deform the $C_{7}$-structure on $W^{n+1}$ by a general position argument.) This means, in particular, that if $X^{n} \cap V_{i}=Q_{i}^{n-8}$ then $X^{n} \cap$ $\left(V_{i} \times c \Sigma_{i}^{7}\right)=Q_{i}^{n-8} \times c \Sigma^{7}$. Moreover, $Q_{i}^{n-8}$ has a collar in $V_{i}^{n-7}$ as does $Q_{i}^{n-8} \times c \Sigma_{i}^{7}$ in $V_{i}^{n-7} \times c \Sigma_{i}^{7}$ and $Q_{i}^{n-8} \times \Sigma_{i}^{7}$ in $V_{i}^{n-7} \times \Sigma_{i}^{7} \subseteq \partial W_{0}^{n+1}$. Calling upon smoothing theory, we may insure that $Q_{i}^{n-8}$ is a smooth submanifold of $V_{i}^{n-7}$ (which inherits a framing by preceding the framing inherited from $V_{i}^{n-7}$ by the inward-pointing normal). This gives rise to a smoothness structure on $Q_{i}^{n-8} \times \Sigma^{7}$ which extends in turn to the smoothness structure on $W_{0}^{n+1}$.

So now, the situation is that $Q_{i}^{n-8}$ is a smooth submanifold of $\partial V_{i}^{n-7}$, framed in its own right, and $Q_{i}^{n-8} \times \Sigma_{i}^{7}$ is a smooth submanifold of $\partial W_{0}^{n+1}$ (with trivial normal bundle). But $X_{0}^{n}=X^{n} \cap W_{0}^{n+1}$ has a collar. Therefore, we may adjust the smoothness structure of $W_{0}^{n+1}$, leaving it untouched on $\bigcup_{i} V_{i} \times \Sigma_{i}^{7}$, so that $X^{n}$ becomes a smooth submanifold of $\partial W_{0}^{n+1}$. We are almost done since there is now an obvious $C_{7}$-structure $M^{n}$ on $X^{n}$, coming from $X_{0}^{n}, Q_{i}^{n-8}$ and the obvious gluing of $Q_{i}^{n-8} \times \Sigma_{i}^{7}$ along part of $\partial X_{i}^{n}$. It is now an easy matter to perform a deformation so that the $C_{7}$-structure on $W_{P L}^{n+1}=I \times X^{n}$ is the product structure $I \times X^{n}$. This is because of the fact that we may first deform the interior of $W_{0}^{n+1}$ so that we may 
think of the $C_{7}$-structure on $W_{P L}^{n+1}$ as of the form $\bar{W}^{n+1} \cup_{\partial} Z^{n+1}$ (or $\bar{W}^{n+1} \cup_{\partial} Z^{n+1}$ if $\partial X^{n}=0$ ) where $\bar{W}_{P L}^{n+1}$ is a small collar neighborhood of $X^{n}$ in $W_{P L}^{n+1}, Z_{P L}^{n+1}$ is its complement and $\bar{W}^{n+1}$ is, essentially, $I \times M^{n}$. We then deform the $C_{7}$-structure on $W_{P L}^{n+1}$ so that $\bar{W}^{n+1}$ "expands" to fill all of $W_{P L}^{n+1}$. Thus Property (I) is verified.

Having constructed $C_{7}$, we may proceed to an inductive definition of $C_{8}$, $C_{9}, \cdots, C_{n}$.

First, we pause here to note that if $Y^{j}$ is a smooth framed manifold and $M^{n}$ is a $C_{7}$-manifold then we may denote by $Y^{j} \times M^{n}$ the obvious $C^{n}$-structure on $Y^{i} \times M_{P L}^{n}$.

Now assume $C_{k-1}$ to have been defined so that for any $C_{k-1}$-manifold $N^{n}$ and any framed smooth manifold $Y^{j}, Y^{j} \times M^{n}$ is defined as a $C_{k}(n+j)$-manifold with $\left(Y^{j} \times M^{n}\right)_{P L}=Y^{j} \times N_{P L}^{n}$. Pick one $C_{k-1}$-structure $\Sigma^{k}$ on $S^{k}$ from each equivalence class.

3.3. Definition. An $n$-dimensional $C_{k}$-manifold is a triple consisting of

(a) A $C_{k-1} n$-manifold $M_{0}^{n}$ where $\partial M_{0}^{n}=R^{n-1} U_{\partial}\left(U_{i} U_{i}^{n-1}\right)$ with the $\left(U_{i}^{n-1}\right)_{P L}$ mutually disjoint.

(b) A set of compact smooth framed manifolds $V_{i}^{n-k-1}$.

(c) A set of $C_{k-1}$ concordances $H_{i}$ between $U_{i}$ and $V_{i}^{n-k-1} \times \Sigma_{i}^{k}$ where $\Sigma_{i}^{k}$ is one of the permitted structures on $S^{k}$.

$M_{P L}^{n}$ is, again, the obvious manifold, viz.

$$
M_{P L}^{n}=\left(M_{0}^{n}\right)_{P L} \cup \bigcup_{i} H_{i} \cup \bigcup_{i}\left(V_{i}^{n-k-1} \times c\left(\Sigma_{i}^{k}\right)\right)_{P L} .
$$

We claim that $C_{k}$ satisfies Axioms (1)-(6) of §2. As before, only Axiom (5) requires any care whatever, and the verification mimics exactly that for the category $C_{7}$. The reader will also easily see that if $Y^{j}$ is a framed smooth manifold and $M^{n}$ a $C_{k}$-manifold, then there is an obvious way to define $Y^{j} \times M^{n}$. Moreover, the proof that $C_{7}$ satisfies Property (I) may be carried over bodily with very minor changes to a proof for $C_{k}$. Thus we state:

\subsection{Corollary. $C_{k}$ satisfies Property (I).}

We next define the manifold-like category $C_{\infty}=\varliminf C_{k}$ as the union of all the manifold-like categories $C_{7} \subseteq C_{8} \subseteq \cdots \subseteq C_{n}$. (We may write inclusion signs inasmuch as any $C_{p}$-manifold obviously acquires a $C_{q}$-structure for $q>p$.) $C_{\infty}$ inherits all the important properties we have discussed thus far; it satisfies Axioms (1)-(6) and is thus a manifold-like category. Moreover, it satisfies Property (I).

We are thus led immediately to

3.5. ThEOREM. $C_{\infty}$ is the PL-manifold category, that is, for each PL-manifold $X^{n}, S_{C_{\infty}}\left(X^{n}\right)$ has precisely one element. 
Proof. The relevant fact is, of course, Lemma 2.3. We have established that $C_{\infty}$ satisfies Property (I); this leaves only Property (II) to be verified and the proof will be complete.

Suppose, then, that $\Sigma^{j}$ is a $C_{\infty}$-manifold with $\Sigma_{P L}^{j} \cong S^{j}$. Then, in particular, $\Sigma^{j}$ is a $C_{k}$-manifold for some $k<j$. But consider the cone on $\Sigma_{P L}^{j}$. This becomes a $C_{j}$-manifold in an obvious way. That is, we form the $C_{j}$-manifold $\Delta^{j+1}$ where $\Delta_{0}^{j+1}=I \times \Sigma^{j}, U^{j}=\iota \Sigma^{j}$ (i.e., $U_{P L}^{j}=\Sigma_{P L}^{j} \times\{1\}$ ), $V$ is a point with the standard framing, and $H$ is also $I \times \Sigma^{j}$, so that $\Delta_{P L}^{j+1}=I \times \Sigma_{P L}^{j} \cup I \times \Sigma_{P L}^{j} U$ $c \Sigma_{P L}^{j}$. (Here the two cylinders $I \times \Sigma_{P L}^{j}$ are laid end-to-end and the free end of the second becomes the base of the cone $c \Sigma_{P L}^{j}$.) Obviously $\Delta_{P L}^{j+1} \cong c S^{j}=D^{j+1}$. Thus Property (II) is verified and the proof of the theorem is complete.

REMARK. As is the case with $C_{7}$, we could define $C_{8}, C_{9}, \cdots$, by merely requiring the "singularities" $V_{i}^{n-k-1}$ of a $C_{k}$-manifold $M^{n}$ to have a smooth structure rather than a smooth, framed structure. Theorem 3.5 would still go through in this case.

4. Classification of $C_{k}$-structures. In this section, we construct a sequence of spaces $B C_{k}$ starting with $B O=B C_{1}=B C_{2}=\cdots=B C_{6}$ and with limit $B P L=$ $B C_{\infty} . B C_{k}$ will play a role in characterizing $S_{C^{k}}\left(X^{n}\right)$ for $P L$-manifolds $X^{n}$ analogous to the role of $B O$ in classical smoothing theory.

We construct $B C_{k}$ by means of Brown's representation theorem for contravariant homotopy functors $[g],[h]$. It should be pointed out that, inasmuch as we are primarily concerned with compact manifolds, we shall ignore distinctions in classifying spaces which cannot be determined by finite complexes. That is, we shall neglect the questions of "phantom" maps and the lim" problem.

Let $K$ be a finite $C W$-complex.

4.1. Definition. A $\left(C_{k}, r\right)$-thickening of $K$ consists of a compact $C_{k}$-manifold $M^{r}$ together with a simple homotopy equivalence $h: K \rightarrow M_{P L}^{r}$. If $\left(M^{r}, f\right)$, $\left(N^{r}, g\right)$ are two $\left(C_{k}, r\right)$-thickenings of $K$, we shall call them equivalent iff there is a $C_{k}$-concordance $W^{r+1}$ between $M^{r}$ and $N^{r}$ so that the diagram

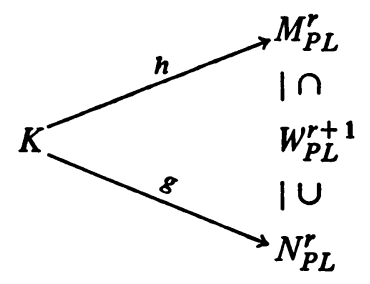

is homotopy commutative.

Denote the set of equivalence classes of $\left(C_{k}, r\right)$-thickenings of $K$ by $T C_{k}^{r}(K)$. We take note that there is an obvious operation $I \times: T C_{k}^{r}(K) \rightarrow T C_{k}^{r+1}(K)$ defined 
by $I \times:\left(M^{r}, f\right) \rightarrow\left(I \times M^{r},(1 / 2) \times f\right)$. In addition, there is a forgetful map $T C_{k}^{r}(K) \rightarrow T C_{k+1}^{r}(K)$. We let $T C_{k}(K)$ be the direct limit of the sequence

$$
\ldots \stackrel{I \times}{\longrightarrow} T C_{k}^{r}(K) \stackrel{I \times}{\longrightarrow} T C_{k}^{r+1}(K) \stackrel{I \times}{\longrightarrow} \cdots .
$$

That is, $T C_{k}(K)$ is the set of stable equivalence classes of $C_{k}$-thickenings of $K$.

4.1. LEMMA. $T C_{k}$ is the restriction to finite $C W$-complexes of a representable homotopy functor to the category of sets.

Proof. We first note that $T C_{k}$ is, in fact, a contravariant homotopy functor from finite $C W$-complexes to sets. For let an element in $T C_{k}(K)$ be represented by $g: K \stackrel{\cong}{\rightarrow} M_{P L}^{r}$ where $M^{r}$ is a $C_{k}$-manifold, and let $f: L \rightarrow K$ be some map where $L$ is a finite $C W$-complex. We may assume without loss of generality that $r$ is very large compared with $\operatorname{dim} L$. Therefore the composition $g \circ f$ may be factored as

$$
L \stackrel{h}{\rightarrow} X^{r} \subseteq M_{P L}^{r}
$$

where $X^{r}$ is a codimension 0 submanifold of $M_{P L}^{r}, h$ is a simple homotopy equivalent. The Regular Neighborhood Axiom A-(5) allows us to put on $X^{r}$ a unique (up to concordance) $C_{r}$-structure, designated $N^{r}$.

We define $f^{*}\left[\left(M^{r}, g\right)\right]=\left[\left(N^{r}, h\right)\right]$ where [ ] denotes equivalence class in $T C_{k}$. One may easily verify that $f^{*}$ is well defined and depends only on the homotopy class of $f$.

Without any difficulty, we may think of the domain of $T C_{k}$ to be pointed finite $C W$-complexes, and we proceed to verify the Wedge Axiom and the MayerVietoris Axiom (cf. [g] , [h], [i]) for $T C_{k}$.

The Wedge Axiom states that if $K=\bigvee_{i=1}^{i} K_{i}$ then the natural map

$$
T C_{k}(K) \rightarrow \prod_{i=1}^{j} T C_{k}\left(K_{i}\right)
$$

is an isomorphism. So let $u \in \Pi_{i} T C_{k}\left(K_{i}\right)$, with the $i$ th coordinate given by $\left[\left(M_{i}^{r}, g_{i}\right)\right]$. (We assume that $r$ is the same for each $i$ and very much larger than $\operatorname{dim} K=\max \operatorname{dim} K_{i}$.) By the Cerf Axiom A-(6), we may assume that $\partial M_{i}^{r}=$ $C_{k}\left(D_{i}^{r-1}\right) \cup_{\partial} P_{i}$ where $D_{i}^{r-1}$ is a copy of the standard smooth $(r-1)$-disc. Pick $j$ disjoint $(r-1)$-discs $E_{i}^{r-1}$ in the boundary of the standard $r$-disc $D_{i}^{r}$. We may identify $E_{i}^{r-1}$ with $D_{i}^{r-1}$ and thus $C\left(D_{i}^{r-1}\right)=C\left(E_{i}^{r-1}\right)={ }^{\prime} C\left(E_{i}^{r-1}\right)$, since $\iota$ is trivial on $C_{k}$-manifolds arising from smooth manifolds. We may then form $\left(\mathrm{U}_{i} M_{i}^{\eta}\right) \cup_{\partial} C\left(D^{\eta}\right)=M^{r}$. Obviously $M_{P L}^{r}=\#\left(M_{i}^{r}\right)_{P L}$ where \# denotes connected sum along boundary. Thus $M_{P L}^{r} \simeq V\left(M_{i}^{r}\right)_{P L} \simeq \bigvee K_{i}=K$. If we denote this homotopy equivalence by $g: K \rightarrow M_{P L}^{r}$, and the inclusion $K_{i} \subseteq V_{i}$ by $\alpha_{n}$ then clearly $\alpha_{n}^{*}\left[\left(M^{r}, g\right)\right]=\left[\left(M_{n}^{r}, g_{n}\right)\right]$. This proves that $T C_{k}(K) \rightarrow \Pi_{i} T C_{k}\left(K_{i}\right)$ is onto. 
To show that the correspondence is one-to-one, observe first, that if $M^{r}$ is concordant in $C_{k}$ to $\bar{M}^{r}$ and $N^{r}$ is concordant to $\bar{N}^{r}$ then $M^{r} \# N^{r}$ is concordant to $\bar{M}^{r} \# \bar{N}^{r}$. Here, we are recalling the Cerf Axiom (6) which both allows us to define \# in $C_{k}$ and suffices to verify the assertion above. Now suppose that $\left(M^{r}, g\right)$ is a $\left(C_{k}, r\right)$-thickening of $K$ where $r$ is very large compared to $\operatorname{dim} K$. The reader may easily check that no generality is lost by assuming that $M^{r}=\# M_{i}^{r}$ where $g$ is the wedge of $g_{i}: K_{i} \stackrel{\cong}{\rightarrow}\left(M_{i}^{r}\right)_{P L}$. Suppose also that $\left(N^{s}, h\right)$ is a $\left(C_{k}, s\right)$ thickening of $K$ with $\left(N^{s}, h\right)=\#\left(N_{i}^{s}, h_{i}\right)$ and $\left[\left(N_{i}^{s}, h_{i}\right)\right]=\left[\left(M_{i}^{r}, g_{i}\right)\right]$ for all $i$. We may assume that $s=r$ and that $\left(N_{i}^{r}, h_{i}\right)$ is actually equivalent to $\left(M_{i}^{r}, g_{i}\right)$ as a $\left(C_{k}, r\right)$-thickening. The first remark of this paragraph concerning connected sums shows that $\left(M^{r}, g\right)$ is equivalent to $\left(N^{r}, h\right)$ as a $\left(C_{k}, r\right)$-thickening, hence as a stable $C_{k}$-thickening. Thus, any element in $T C_{k}\left(\bigvee_{i} K_{i}\right)$ is determined by its image in $\Pi_{i} T C_{k}\left(K_{i}\right)$. Thus the Wedge Axiom holds.

As for the Mayer-Vietoris Axiom, this states that if, with relation to the diagram

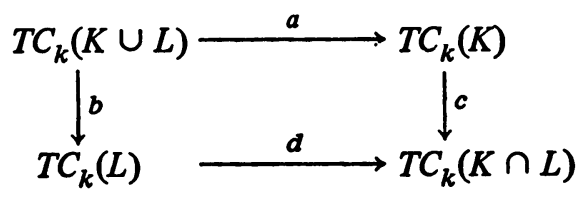

there are elements $u \in T C_{k}(K), v \in T C_{k}(L)$ with $c u=d v$, then there is an element $w \in T C_{k}(K \cup L)$ such that $a w=u, b w=v$.

To prove this, let $\left(M^{r}, g\right)$ represent $u$ and $\left(N^{r}, h\right)$ represent $v$. We may assume that $g, h$ embed $K \cap L$ in $\partial M_{P L}^{r}, \partial N_{P L}^{r}$ respectively. By Axiom A-(5), the regular neighborhood $X^{r-1}$ of $K \cap L$ in $\partial M_{P L}^{r}$ acquires a $C_{k}$-structure $Q^{r-1}$. Similarly, the regular neighborhood $Y^{r-1}$ of $K \cap L$ in $\partial N_{P L}^{r}$ acquires a $C_{k}$-structure $R^{-1}$. Without loss of generality, we may assume that the $\left(C_{k}, r-1\right)$ thickenings $\left(Q^{r-1}, g \mid(K \cap L)\right),\left(R^{r-1}, h \mid(K \cap L)\right)$ are $\left(C_{k}, r-1\right)$ equivalent via a concordance $W^{r}$. Form the $C_{k}$-manifold $P^{r}=M^{r} \cup_{\partial} W^{r} \cup_{\underline{\partial}} N^{r}$, the union attaching $Q^{r-1}$ and $R^{r-1}$ to either end of $W^{r}$. There is an obvious homotopy equivalence $f: K \cup L \rightarrow P_{P L}^{r}$ and clearly, if $w=\left[\left(P^{r}, f\right)\right]$ then

$$
a w=\left[\left(M^{r}, g\right)\right]=u, \quad b w=\left[\left(N^{r}, h\right)\right]=v .
$$

We also need the following countability condition:

4.2. Proposition. $T C_{k}\left(S^{n}\right)$ is countable.

This will follow from

4.3. Lemma. Let $X^{n}$ be a PL-manifold and let $Q^{n-1}$ be a $C_{k}$-manifold with $Q_{P L}^{n-1}$ a codimension 0 submanifold of $\partial X^{n}$. Then, up to concordance, there are only countably many $C_{k}$-manifolds $M^{n}$ with $M_{P L}^{n}=X^{n}$ and $\partial M^{n}=$ $P^{n-1} \cup_{\partial} Q^{n-1}$. 
Proof OF LEMMA. The proof proceeds by induction on $k$, exploiting the fact that classical smoothing theory tells us the result is true for $C_{k}=$ smooth manifolds, i. e., $k \leqslant 6$. Let us consider, therefore, a $P L$-manifold $X^{n}$ such that the $C_{k}$-manifold $Q^{n-1}$ has $Q_{P L}^{n-1}$ a codimension 0 submanifold of $\partial X^{n}$. We must show that there are at most countably many ways of extending this $C_{k}$ structure to all of $X^{n}$. First of all, note that the specification of a $C_{k}$-structure on a $P L$-manifold $Y^{n}$ involves the selection of a codimension $(k+1)$ framed submanifold. That is, the smooth manifold which is the "singularity", away from which the $C_{k}$-manifold is $C_{k-1}$, is, in an obvious way, a framed submanifold of the underlying $P L$-manifold. Thus, in extending the $C_{k}$-structure on $Q_{P L}^{n-1}$ we first have to extend some framed submanifold $W^{n-k-2}$ of $Q_{P L}^{n-1}$ to a framed submanifold $V^{n-k-1}$ on $X^{n}$. We may think of framed submanifolds of $X^{n}$ as simplicial maps of some compatible triangulation of $X^{n}$ to the sphere $S^{k+1}$ (the manifold is the inverse image of the barycenter of a $(k+1)$-simplex of $\left.S^{k+1}\right)$, and thus we may think of the extension of the framed submanifold $W$ to a framed submanifold $V$ as a simplicial extension to $X^{n}$ of a simplicial map $Q_{P L}^{n-1} \rightarrow S^{k+1}$. Obviously, there are at most countably many such extensions. Next, the $C_{k}$-structure on $Q^{n-1}$ involves a smooth, framed structure on $W^{n-k-2}$. (Framing in this case means a smooth framing of the stable smooth normal bundle determined by the choice of smoothing of $W$.) This structure must be extended to $V$. Up to concordance, there are only countably many ways to do this. Next, we note that for each component $W_{i}$ of $W^{n-k-2}$, we have picked a $C_{k-1}$-structure $\Sigma_{i}^{k}$ on the "linking" sphere of $W_{i}$ in $Q_{P L}^{n-1}$. We must extend this choice to the components of $V^{n-k-1}$ (where it is not already determined by what happens to the $W_{i}$ ). The inductive hypothesis tells us that there are only countably many concordance classes of $C_{k-1}$-structures on $S^{k}$, and thus, since we may use only one $\Sigma^{k}$ from each concordance class, there are only countably many choices that have to be made.

The effect of all the choices we have made thus far is to give us a $C_{k-1}$ structure on $\left(V \times S^{k}\right) \cup\left(Q_{P L}^{n-1}-\left(W \times D^{k+1}\right)\right)$. We now must extend this to a $C_{k-1}$-structure on the rest of $X^{n}-\left(V \times D^{k+1}\right)$. But, by the induction hypothesis, there are, up to concordance, only countably many ways of doing this.

To summarize then, any concordance class of $C_{k}$-structures on $X^{n}$ extending $Q^{n-1}$ will be determined by one of a countable number of possible choices from a certain set of invariants. This proves Lemma 4.3. Proposition 4.2 follows immediately.

We now extend the homotopy functor $T C_{k}$ from finite $C W$-complexes to all $C W$-complexes. We do this in the crudest possible way, i.e.,

$$
T C_{k}(K)=\lim T C_{k}\left(K_{i}\right)
$$


where $K_{i}$ ranges over all finite subcomplexes. Brown's theorem enables us to assert that the functor $T C_{k}$ has a classifying space $B C_{k}$. This completes the proof of the theorem.

REMARK. We may talk about a sequence of "maps"

$$
B O \rightarrow B C_{7} \rightarrow B C_{8} \rightarrow \cdots \rightarrow B P L .
$$

The reason for the quotation marks is that the maps $B O \rightarrow B C_{k}$ and $B C_{k} \rightarrow$ $B P L$ may not be quite honest inasmuch as they are only truly realized on finite subcomplexes of their respective domains. However, since our primary concern is with finite complexes, i. e., compact $C_{k}$-manifolds, ignoring this nicety causes no problems.

REMARK. As we have been observing all along, we could have obtained the result above if we had defined $C_{k}$-manifolds by allowing singularities of $C_{k-1}$. manifolds along smooth manifolds, rather than insisting upon smooth framed manifolds. However, the next result gives a clear motivation for the more exacting definition.

4.4. THEOREM. $B C_{k}$ has the homotopy type of $B C_{k-1}$ with $(k+1)$ cells adjoined. In particular, there will be one $(k+1)$-cell for each element in $S_{C_{k-1}}\left(S^{k}\right)$.

Proof. We are really only concerned with finite approximations to $B C_{k-1}$, $B C_{k}$, etc., and so we shall prove the result in that context.

Therefore, let $K, L$ be a finite pair approximating $B C_{k}, B C_{k-1}$. If we wish, we may think of $K$ as a high-dimensional $P L$-manifold with a certain $C_{k}$-structure; $L$ is a codimension 0 submanifold of $K$ which misses the highest order singularities, so that its $C_{k}$-structure is effectively a $C_{k-1}$-structure. By the definition of $C_{k}$ manifold we see that $K$ has the form $\left(\cup_{i} V_{i} \times c \Sigma_{i}^{k}\right) \cup K_{0}$ where $K_{0}$ has a certain $C_{k-1}$-structure. Since $L$, with its $C_{k-1}$-structure, is a codimension 0 submanifold of $K_{0}$, it follows that $K_{0}$ is an even better finite approximation to $B C_{k-1}$ than $L$ is. We therefore may as well assume that $L=K_{0}$. Assume now that the $V_{i}$ are indexed so that $\Sigma_{i}^{k}=\Sigma_{j}^{k}$ if and only if $i=j$. That is, we consider together as one manifold all the pieces of the singular part which have the same $C_{k-1}$-sphere as link. Let $n$ be the dimension of $K$ viewed as a manifold. We claim that $I \times K^{n}$ may be embedded as a codimension 0 submanifold of a $C_{k}$ manifold $\bar{K}^{n+1}$ such that the singular parts $\bar{V}_{i}^{n-k}$ of $\bar{K}^{n+1}$ are at least $([(n-k) / 2]-1)$-connected. To see this do surgery starting from one end $1 \times V_{i}$ of $I \times V_{i}$ so that the result is $([(n-k) / 2]-1)$-connected and is still framed. Call the result $\bar{V}_{i}^{n-k}$. Let $H_{i}$ denote a collar $\left(I \times \Sigma_{i}^{k}\right)$. Then it is easily seen that we 
may assume that $\left(I \times V_{i}\right) \times\left(c \Sigma_{i}^{k} \cup H_{i}\right)$ intersects $I \times K_{0}$ in a "collar neighborhood" $\left(I \times V_{i}\right) \times H_{i}$. If we set $W_{i}=$ closure $\left(\bar{V}_{i}-\left(I \times V_{i}\right)\right)$, we can form the $C_{k}$-manifold $P^{n+1}=W_{i} \times\left(c \Sigma_{i}^{k} \cup H_{i}\right)$ whose $C_{k}$-structure is obvious. We then set $\bar{K}^{n+1}=\left(I \times K^{n}\right) \cup_{\partial} P^{n+1}$.

It is obvious that $\left(\bar{K}, K_{0}\right)$ is an even better approximation of $\left(B C_{k}, B C_{k-1}\right)$ than $K, K_{0}$. So we state the following paradigm. $B C_{k}$ may be approximated arbitrarily well by a $C_{k}$-manifold $K^{n}$ whose nonsingular (i.e., $C_{k-1}$ ) part $K_{0}^{n}$ is an arbitrarily good approximation of $B C_{k-1}$, and such that each component $V_{i}^{n-k-1}$ of the singular part is arbitrarily highly connected (since $n$ is arbitrarily high). Since $V_{i}^{n-k-1}$ is arbitrarily close to being contractible, $V_{i}^{n-k-1} \times \Sigma_{i}^{k}=$ $K_{0}^{n} \cap\left(V_{i}^{n-k-1} \times c \Sigma_{i}^{k}\right)$ is arbitrarily close to the homotopy type of $S^{k}$. We leave it to the reader to show that we may force the approximating $C_{k}$-manifold $K^{n}$ to have $V_{i}$ 's corresponding to any finite subset of $S_{C_{k-1}}\left(S^{k}\right)$. It follows from the foregoing that to approximate $B C_{k}$ arbitrarily well, we need only consider $K_{0} \cup\left(p_{i} \times c \Sigma_{i}^{k}\right)$ where $p_{i} \in V_{i}$. That is, to approximate $B C_{k}$, we approximate $B C_{k-1}$ and then add $(k+1)$-cells. This proves the theorem.

REMARK. If $e_{i}^{k+1}$ is a cell of $B C_{k} \bmod B C_{k-1}$ corresponding to [ $\left.\Sigma_{i}^{k}\right] \in$ $S_{C_{k-1}}\left(S^{k}\right)$, then the attaching map is the map $S^{k} \rightarrow B C_{k-1}$ classifying the $C_{k-1}$ thickening of $S^{k}$ determined by $\left(\Sigma_{i}^{k}\right)_{P L} \stackrel{\cong}{\rightarrow} S^{k}$.

4.5. Proposition. Let $X^{n}$ be a PL-manifold; $S_{C_{k}}\left(X^{n}\right)$ is in $1-1$ correspondence with homotopy classes of liftings

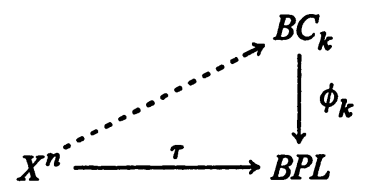

where $\tau$ classifies the stable tangent bundle of $X^{n}$ and $\phi_{k}$ is the natural map.

This follows from results of Rourke [j], or the reader may prove it directly.

4.6. Proposition. The map $\phi_{k}$ is $(k+1)$-connected.

Proof. By the results of $\S 3$, any $P L n$-thickening of a finite complex $K^{j}$ has a $C_{r}$-structure for large enough $r$. We claim that if $j<r+1$ the $P L n$-thickening may be given a $C_{r-1}$-structure. For if $h: K^{j} \rightarrow X^{n}$ is the $P L$-thickening, $X^{n}$ has a $j$-dimensional spine (which we may assume to contain $\operatorname{im} h$ ). The $C_{r}$ structure on $X^{n}$ gives a $C_{r-1}$-structure on the complement of a tubular neighborhood of a submanifold of codimension $r+1$. We may assume, therefore, that the spine of $X^{n}$ misses this tubular neighborhood, by general position considerations. So let $\bar{X}^{n}$ be a small regular neighborhood of the spine lying wholly within the $C_{r-1}$ part of $X^{n}$. By the Regular Neighborhood Axiom (5), $\bar{X}^{n}$ acquires a 
$C_{r-1}$-structure. But the thickening of $K^{j}$ into $\bar{X}^{n}$ is $P L$-equivalent to the original thickening into $X^{n}$. By induction, we then see that any $P L$-thickening of a $j$-dimensional complex pulls back to a $C_{k}$-thickening of $j \leqslant k+1$. Since BPL classifies stable $P L$-thickenings $T P L$, it is obvious that $\phi_{k}: B C_{k} \rightarrow B P L$ induces an epimorphism of homotopy groups in dimensions up to $k+1$. A relative version of the same argument shows that $\phi_{k}$ induces a monomorphism in dimensions up to $k$. Thus the proposition is proved.

5. The homology groups of $(B P L, B O)$. The purpose of this section is to make a few points about the integral homology groups $H_{*}(B P L, B O)$ in the light of the foregoing results. As a first step, consider the fact that Theorem $4.4 \mathrm{im}$ mediately implies that we may think of $H_{*}(B P L, B O)$ as the homology of a chain complex $\Gamma_{*}$ whose generators in dimension $k+1$ are in 1-1 correspondence with $S_{C_{k-1}}\left(S^{k}\right)$. We wish to characterize the boundary operator $\delta$ of $\Gamma_{*}$.

A typical generator of $\Gamma_{k+1}$ is a $k$-sphere $\Sigma^{k}$ with a $C_{k-1}$-structure. This structure involves a multiply-punctured sphere $\Sigma_{0}^{k}$ with a $C_{k-2}$-structure, together with a identification of each boundary component of $\Sigma_{0}^{k}$ with a $C_{k-2}$-structure on the $(k-1)$-sphere of the form $p \times \Sigma^{k-1}$, where $p$ is a framed point and $\Sigma^{k-1}$ is the $C_{k-2}$-structure chosen to represent its concordance class. Thus, each boundary component of $\Sigma_{0}^{k}$ is associated to the appropriate $\Sigma^{k-1}$. (N. B.: This association does not depend on the framing of the point.) Moreover, since $p \times$ $\Sigma^{k-1}$ is thought of as a structure on the standard sphere, $\left(p \times \Sigma^{k-1}\right)_{P L}$ inherits an orientation. On the other hand, $\left(\Sigma_{0}^{k}\right)_{P L}$ is a codimension zero submanifold of the standard $k$-sphere, therefore it acquires an orientation, as do all the boundary components. Thus, depending on how a given boundary component is identified with $p \times \Sigma^{k-1}$, the two orientations may differ or agree. In the former case, call the boundary component positive; in the latter, negative. Now, given $\Sigma^{k}$, if $\left\{\partial_{i} \Sigma_{0}^{k}\right\}$ are the distinct boundary components, define $\delta \Sigma^{k}=\sum \pm \Sigma_{i}^{k-1}$, where $\partial_{i} \Sigma_{0}^{k}$ is identified with $p \times \Sigma_{i}^{k-1}$ and where the sign is positive or negative according as the boundary component is positive or negative. We leave it to the reader to check that $\delta^{2}=0$.

Now note that a smooth sphere $\Sigma^{k}$ is naturally a $C_{k-1}$-sphere. Moreover, it is obvious that $\delta \Sigma^{k}=0$.

5.1. Proposition. Let the smooth sphere $\Sigma^{k}$ correspond to the element $\alpha \in \pi_{k}(P L / O) \cong \pi_{k+1}(B P L, B O)$; then the cycle $\Sigma^{k}$ of $\Gamma_{k+1}$ represents the homology class in $H_{k+1}(B P L, B O)$ which is the image of $\alpha$ under the Hurewicz homomorphism.

The proof is left to the reader 
6. Some spectral sequences. At this point, we append some remarks concerning the relation between the categories we have constructed and bordism. Let $D_{m, n}$ be defined as follows: Consider $C_{m}$-manifolds $M^{m+n+1}$ with smooth boundary. Call $M^{m+n+1}$ equivalent to $N^{m+n+1}$ iff there is a $C_{m}$-manifold $W^{m+n+2}$ with $\partial W^{m+n+2}=\left(M^{m+n+1} \amalg \iota N^{m+n+1}\right) \cup_{\partial} V^{m+n+1}$ where $V^{m+n+1}$ is smooth. Equivalence classes of such manifolds form a group $D_{m, n}$ under disjoint union.

Set $E_{m, n}=\bigoplus_{\alpha}\left(\Pi_{n}^{s}\right)_{\alpha}$ where $\Pi_{n}^{s}$ denotes the group of framed bordism classes of framed manifolds and where $\alpha$ ranges over the elements of $S_{C_{m-1}}\left(S^{m}\right)$.

There is an obvious map $i: D_{m-1, n+1} \rightarrow D_{m, n}$. We can define a map $j: D_{m, n} \rightarrow E_{m, n}$ as follows: A $C_{m}(m+n+1)$-manifold $M^{m+n+1}$ has a "singular set" consisting of disjoint smooth manifolds $V_{i}^{n}$, each conceived of as a framed manifold, and each having a $C_{m-1} m$-sphere $\Sigma_{i}^{m}$ as link. If we consider all the $n$-manifolds with the same link as one manifold (i.e., the disjoint union), we can define $j$ on the equivalence class of $M^{m+n+1}$ as having $\left[V_{i}^{n}\right]$ as the $\Sigma_{i}^{m}$ th coordinate, the $\alpha$ coordinate being zero if $\alpha$ is not among the $\Sigma_{i}^{m}$. We leave it to the reader to verify that $j$ is well defined.

We also define $k: E_{m, n} \rightarrow D_{m-1, n}$ by taking $k\left[V_{\alpha}^{n}\right]=\left[\amalg_{\alpha} V_{\alpha}^{n} \times \Sigma_{\alpha}^{m}\right]$ where [ ] on the right-hand side denotes equivalence class in $D_{m-1, n}$. (Note that each product $V_{\alpha}^{n} \times \Sigma_{\alpha}^{m}$ has a $C_{m-1}$-structure.) Again, verification that $k$ is well defined is routine.

If we let $D=\Sigma_{m, n} D_{m, n}, E=\Sigma_{m, n} E_{m, n}$ it is also routine to see that

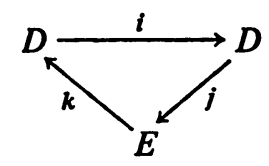

is an exact couple.

6.1. Proposition. The spectral sequence of the exact couple (1) converges to $E_{m, n}^{\infty}$ where $\Sigma_{m+n=p} E_{m, n}^{\infty}$ is a graded object associated to a filtration of the group $\mathrm{N}_{\rho+1}^{\mathrm{rel}}=$ group of bordism classes of PL-manifolds with smooth boundary.

This is easily seen since (1) is a regular $\partial$-couple $[\mathrm{k}]$, and $D_{p+1,-1}$ is easily seen to be $N_{p+r}^{\text {rel }}$.

In the last section, we introduced a crain complex $\Gamma_{*}$, where $\Gamma_{m+1}=$ free abelian group on $S_{C_{m-1}}\left(S^{m}\right)$. We now introduce $\Delta_{*}$, a new chain complex where $\Delta_{m+1}=\Gamma_{m+1}$ but where the boundary operator $\epsilon$ has a different meaning. Let $\Sigma^{m}$ be a generator of $\Delta_{m+1}$. Then the boundary components of the nonsingular part of $\Sigma^{m}$ each are associated with a $C_{m-2}(m-1)$-sphere $\Sigma_{i}^{m-1}$. The singular point $p_{i}$ with link $\Sigma_{i}^{m-1}$ is thought of as a framed, i.e., oriented, point. Let 
$\epsilon \Sigma^{m}=\sum \pm \Sigma_{i}^{m-1}$, with sign determined according to whether $p_{i}$ is positively or negatively oriented.

We may now easily prove

6.2. COROLLARY. The $E^{2}$ term of the spectral sequence of (1) is given by $E_{m, n}^{2}=H_{m+1}\left(\Delta_{*} \otimes \Pi_{n}^{s}\right)$. $\otimes \Pi_{n}^{s}$.

We leave to the reader details of the proof, noting only that $E_{m, n}=\Gamma_{m+1}$

We may remark that, in the construction of the manifold-like categories $C_{k}$, we could have dropped the condition that singular sets be framed smooth manifolds, requiring merely that they be smooth. Call the manifold-like categories constructed in this fashion $\bar{C}_{k}$; again $B \bar{C}_{k}$ exist and $\lim _{\longrightarrow} B \bar{C}_{k}=B P L$. (However, this is not a skeletal filtration.) Let $\bar{\Gamma}^{*}$ be constructed as a chain complex in analogy to $\Gamma_{*}$.

Set $\bar{D}_{m, n}$ to be the group defined like $D_{m, n}$ but with $C_{m}$ replaced with $\bar{C}_{m}$ throughout. Likewise define $\bar{E}_{m, n}$ as $\bigoplus_{\alpha}\left(N_{n}\right)_{\alpha}$ where $N_{n}$ denotes smooth bordism and $\alpha$ ranges over $S_{\bar{C}_{m-1}}\left(S^{m}\right)$.

In addition set $D_{m, n}^{\prime}$ to be defined like $\bar{D}_{m, n}$ with "manifold" replaced by "oriented manifold" and bordisms required to be oriented. Set $E_{m, n}^{\prime}=\bigoplus_{\alpha}\left(\Omega_{n}\right)_{\alpha}$ where $\Omega_{n}$ denotes oriented smooth bordism and $\alpha$ again ranges over $S_{\bar{C}_{m-1}}\left(S^{m}\right)$. Then we have exact couples
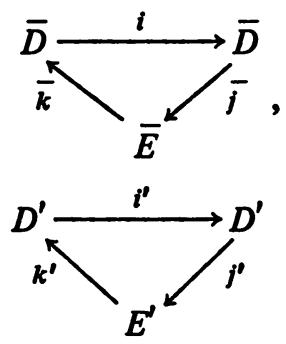

6.3. COROLlaRY. In the spectral sequence of (2) (resp. (3)), $\Sigma_{m+n=p} E_{m, n}^{\infty}$ is a graded object associated to $N_{p+1}^{\mathrm{rel}}\left(\right.$ resp. $\left.\Omega_{p+1}^{\mathrm{rel}}\right)$. In addition, $E_{m, n}^{2}=$ $H_{m+1}\left(\bar{\Gamma}_{*} \otimes N_{n}\right)\left(\right.$ resp. $\left.H_{m+1}\left(\bar{\Gamma}_{*} \otimes \Omega_{n}\right)\right)$.

In point of fact, the spectral sequences of (1), (2), (3) are thinly-disguised versions of spectral sequences arising from filtrations of $M P L \bmod M O$ (in the case of (1) and (2)) and MSPL mod MSO (in the case of (3)). In (1) the filtration of $M P L$ comes from that of the base space $B P L$ via $B O \subseteq B C_{1} \subseteq \cdots \subseteq B P L$. In (2), the relevant filtration is

and in (3),

$$
B O \subseteq B \bar{C}_{1} \cdots B \bar{C}_{k} \subseteq B P L
$$

$$
B S O \subseteq \widetilde{B \bar{C}_{1}} \cdots \widetilde{B \bar{C}_{k}} \cdots B S P L
$$

where $\sim$ denotes 2 -fold covering by the orientation sheaf. 


\section{REFERENCES}

[a] D. Sullivan, Notes on geometric topology, M. I. T., 1970 (mimeographed notes).

[b] N. Baas, On bordism theory of manifolds with singularities, Mimeographed notes, Aarhus, 1970.

[c] M. W. Hirsch and B. Mazur, Smoothing of PL-manifolds. I, II, Ann. of Math. Studies, Princeton Univ. Press, Princeton, N. J. (to appear).

[d] - Smoothings of PL-manifolds, Cambridge University Topology Seminar (mimeographed notes).

[e] J. F. P. Hudson, Piecewise linear topology, Univ. of Chicago Lecture Notes, Benjamin, New York, 1969. MR 40 \#2094.

[f] M. A. Kervaire and J.W. Milnor, Groups of homotopy spheres. I, Ann. of Math. (2) 77 (1963), 504-537. MR 26 \#5584.

[8] E. H. Brown, Jr., Cohomology theories, Ann. of Math. (2) 75 (1962), 467-484. MR 25 \#1551.

[h] E. H. Spanier, Algebraic topology, McGraw-Hill, New York, 1966. MR 35 \#1007.

[i] J. F. Adams, $A$ variant of $E$. $H$. Brown's representability theorem, Topology 10 (1971), 185-198.

[j] C. P. Rourke, On structure theorems (mimeographed notes).

[k] S. T. Hu, Homotopy theory, Pure and Appl. Math., vol. 8, Academic Press, New York, 1959. MR 21 \#5186.

DEPARTMENT OF MATHEMATICS, RUTGERS COLLEGE, UNIVERSITY HEIGHTS CAMPUS, NEW BRUNSWICK, NEW JERSEY 08903 